\title{
Ingested plant miRNAs regulate gene expression in animals
}

\author{
Hervé Vaucheret $^{1}$, Yves Chupeau ${ }^{1}$ \\ ${ }^{1}$ Institut Jean-Pierre Bourgin, INRA, 78000 Versailles, France \\ Cell Research (2012) 22:3-5. doi:10.1038/cr.2011.164; published online 25 October 2011
}

The incidence of genetic material or epigenetic information transferred from one organism to another is an important biological question. A recent study demonstrated that plant small RNAs acquired orally through food intake directly influence gene expression in animals after migration through the plasma and delivery to specific organs.

Non-protein coding RNAs, and in particular small RNAs, were recently revealed as master chief regulators of gene expression in all organisms. Endogenous small RNAs come in different flavors, depending on their mode of biogenesis. Most microRNAs (miRNA) and short interferring RNAs (siRNA) derive from long double-stranded RNA (dsRNA) precursors that are processed into small RNA duplexes, 20 to 25nt long, by RNaseIII enzymes called Dicer [1]. One strand of small RNA duplexes is loaded onto an Argonaute protein that executes silencing by cleaving or repressing the translation of homologous mRNA [2]. In certain species, RNA cleavage is followed by DNA methylation and/or histone modification, leading to heritable epigenetic modification [3].

Endogenous small RNAs play essential roles during development and stress responses, and control transposable

Correspondence: Hervé Vaucheret

E-mail: herve.vaucheret@versailles.inra.fr elements and chromatin states. Small RNAs can also be produced in response to invasion by exogenous nucleic acids from viruses, bacteria, transgenes, etc [4]. Under these circumstances, small RNAs act in defense mechanisms by directing the destruction of the invader. Importantly, exogenous small RNAs and some endogenous small RNAs are mobile within certain organisms [5]. Moreover, exogenous small RNAs can be amplified during the defense mechanisms, allowing the spreading of RNA silencing from the cell where it is activated to the rest of the organism [6].

Immediately after its discovery, the potent effect of small RNAs has been exploited to specifically downregulate gene expression in a timely controlled manner. Such technology is commonly used in the laboratory but also for biomedical applications. Indeed, artificially synthesized small RNAs or dsRNA can be introduced exogenously to look for transient and localized effects. Artificial small RNAs can also be expressed durably from stably integrated transgenes or using replicating viruses. In certain organisms, a simple and convenient manner to induce RNA silencing consists in feeding the recipient host with bacteria expressing dsRNA homologous to the cognate target. This method works with worms and paramecia [7, 8]. Small RNAs or dsRNAs can also be transferred from plant to pests such as insects that eat leaves or nematodes that infect roots. Indeed, transgenic plants expressing
dsRNA homologous to essential genes of insect pests or nematodes specifically resist these parasites due to the silencing activity of small RNAs expressed from the plant transgenes [9-11].

Given that human food relies largely on plants, it was expected that researchers would look for the outcome of plant small RNAs after food intake, and for a potential effect of the ingested plant small RNAs on the expression of human genes. In the paper by Zhang et al. [12] in this issue of Cell Research, cloning and sequencing of small RNAs in human serum revealed that plant miRNAs represented about $5 \%$ of mammalian miRNAs. Plant miRNAs are 2'-O-methyl modified at their $3^{\prime}$ end, which renders them resistant to periodate, whereas human miRNAs have free $2^{\prime}$ and $3^{\prime}$ hydroxyl, which renders them sensitive to periodate. The plant miRNAs cloned from human serum were resistant to periodate, indicating that they are genuine plant miRNAs, probably coming from the food intake. Confirming this hypothesis, the concentration of plant miRNAs was higher in the serum of rice-fed mice compared with chow diet-fed mice. Moreover, adding plant miRNAs to chow diet resulted in an increase of plant miRNA concentration in mouse serum. Interestingly, cooking did not impair the accumulation of plant miRNAs, indicating that they are resistant to heat and thus could be acquired from both raw and cooked meals.

Plant miRNAs were primarily de- 
tected in plasma microvesicles (MV) [12]. MVs are shed from almost all cell types and have the potential to selectively interact with specific target cells and mediate intercellular communication by transporting lipids, RNA and proteins. Consistently, plant miRNAs were detected in various tissues, including liver, intestine and lung. Different plant miRNAs accumulated at different levels, which also varied from one tissue to another, but their levels could reach up to one tenth of the most abundant human miRNA. Indeed, human miRNAs have a dynamic range of accumulation, extending from 1 copy to $>10000$ copies per cell. The amount of the most abundant plant miRNA found in human serum, miR168, was $3.2 \times$ $10^{-6} \mathrm{fmol}$ (1 920 copies) per $100 \mathrm{pg}$ of total RNA, equivalent to 850 copies per cell, which is equivalent to the average amount of a human miRNA.

Plant miR168 is one of the most important miRNA in plants. Indeed, it regulates $A G O 1$ mRNA, which encodes the core component of the plant RNA silencing complex. As such, AGO1 binds to the vast majority of plant miRNAs. Regulating $A G O 1$ by miR168 ensures a feedback loop that allows adjusting the level of AGO1 to the amount of miRNAs in the cell. AGO1 also binds to exogenous siRNAs induced by defense mechanisms. Exogenous siRNAs competes with miRNAs and deplace the AGO1/miR168 equilibrium to adjust the amount of AGO1 to the amount of exogenous siRNAs required for the plant defenses [13].

Unexpectedly, plant miR 168 exhibits a high degree of complementarity with the exon 4 of mammalian LDLRAP1 (Low Densitiy Lipoprotein Receptor Adapter Protein 1) mRNA [12]. $L D L-$ $R A P 1$ is a liver-enriched mRNA encoding a protein that facilitates the removal of LDL (Low Density Lipoprotein) from the circulatory system. The level of LDLRAP1 protein but not $L D L R A P 1$ mRNA was decreased in rice-fed mice compared with chow diet-fed mice, indicating that plant miR168 executes silencing like a human miRNA in human cells. The effect of miR168 was sequence specific because adding the LDLRAP1 complementarity sequence to a luciferase reporter resulted in a decrease of luciferase activity in cells cotransfected with miR168. In addition, mutating the LDLRAP1 complementarity sequence abolished the silencing effect of miR168.

The authors of this study hypothesized that epithelial cells in the intestine might take up miRNAs in food, package them into MVs and release them into the circulatory sytem [12]. The secreted MVs could then deliver exogenous plant miRNA to target organs where they could regulate cognate mRNAs. They tested this hypothesis by transfecting human intestinal epithelial Caco-2 cells with plant miR168, and used the MVs released by the Caco- 2 cells to treat HepG2 cells. Immunoprecipitation revealed that miR168 and LDLRAPI mRNA associate with human RNA silencing complex in HepG2 cells treated with Caco-2 MVs transfected with miR168. Eventually, LDLRAP1 protein level was decreased in HepG2 cells, indicating that MVs carried a functional miR168 to specific organs.

Downregulation of LDLRAP1 in the liver causes decreased endocytosis of LDL by liver cells and impairs removal of LDL from the plasma [12]. Consistent with the silencing effect of plant miR168 on LDLRAP1 protein, LDL levels in mouse plasma were elevated following miR168 uptake. This effect was specific to miR168 because injection of an anti-miR168 oligonucleotide blocked the increase in LDL levels.

These results raise the question of whether food-derived small RNAs could play an active role in human/ animal health. This question is valid for both plant miRNAs and animal miRNAs. Indeed, this study also revealed that adding an animal miRNA, miR-150, to the mouse diet, allows the increase of miR-150 levels in the liver and downregulation of its natural target c-Myb [12]. Human diets are extremely diverse and rely on various amounts and various species of plants and animals in different parts of the world. Whether physiological and/or pathological differences induced by the foods could be partly determined by the small RNA repertoire of each diet remains to be determined.

Plants encode hundreds of thousands of different small RNAs [14]. Given that six nucleotides of perfect complementary between the "seed" region of a small RNA and its target is sufficient to promote RNA silencing in mammals [15], how many plant miRNAs have the potential to actually regulate gene expression in animals? One could speculate that biologically active plant miRNAs in our diet are orders of magnitude lower than biologically active animal miRNAs that are ingested when eating meat. This issue will certainly be addressed in the near future. Given the stability of miRNAs in the gut, their influence on the bacterial community is yet another field of interrogation.

During the last century, the outcome of proteins has been examined, mostly due to the extension of bovine spongiform encephalopathy, a disease caused by prions (a class of degenerative proteins), which raised important concerns in animal farming and human nutrition. After the release of genetically modified organisms (GMO) on the market, the fate of trangenes and transgene products in the digestive tract have also been questioned. With this study, the possible incidence of RNA contained in the food diet on animal/human health will certainly become an explosive field of investigation. This study also implies precise awareness from biotechnologists who intend to make use of dsRNA, especially in the field of plant protection against pests.

\section{References}

1 Carthew RW, Sontheimer EJ. Origins and mechanisms of miRNAs and siR- 
NAs. Cell 2009; 136:642-655.

2 Bartel DP. MicroRNAs: target recognition and regulatory functions. Cell 2009; 136:215-233.

3 Law JA, Jacobsen SE. Establishing, maintaining and modifying DNA methylation patterns in plants and animals. Nat Rev Genet 2010; 11:204-220.

4 Ding SW, Voinnet O. Antiviral immunity directed by small RNAs. Cell 2007; 130:413-426.

5 Molnar A, Melnyk CW, Bassett A, Hardcastle TJ, Dunn R, Baulcombe DC. Small silencing RNAs in plants are mobile and direct epigenetic modification in recipient cells. Science 2010; 328:872-875.

6 Palauqui JC, Elmayan T, Pollien JM, Vaucheret H. Systemic acquired silencing: transgene-specific post-transcriptional silencing is transmitted by grafting from silenced stocks to non-silenced scions. EMBO J 1997; 16:4738-
4745 .

7 Galvani A, Sperling L. RNA interference by feeding in Paramecium. Trends Genet 2002; 18:11-12.

8 Timmons L, Court DL, Fire A. Ingestion of bacterially expressed dsRNAs can produce specific and potent genetic interference in Caenorhabditis elegans. Gene 2001; 263:103-112.

9 Baum JA, Bogaert T, Clinton W, et al. Control of coleopteran insect pests through RNA interference. Nat Biotechnol 2007; 25:1322-1326.

10 Fairbairn DJ, Cavallaro AS, Bernard M, Mahalinga-Iyer J, Graham MW, Botella JR. Host-delivered RNAi: an effective strategy to silence genes in plant parasitic nematodes. Planta 2007; 226:1525-1533.

11 Mao YB, Cai WJ, Wang JW, et al. Silencing a cotton bollworm P450 monooxygenase gene by plant-mediated RNAi impairs larval tolerance of gos- sypol. Nat Biotechnol 2007; 25:13071313.

12 Zhang L, Hou D, Chen X, et al. Exogenous plant MIR168a specifically targets mammalian LDLRAP1: evidence of cross-kingdom regulation by $\mathrm{mi}$ croRNA. Cell Res 2012; 22:107-126.

13 Martinez de Alba AE, Jauvion V, Mallory AC, Bouteiller N, Vaucheret $\mathrm{H}$. The miRNA pathway limits AGO1 availability during siRNA-mediated PTGS defense against exogenous RNA. $\mathrm{Nu}$ cleic Acids Res 2011; 39:9339-9344.

14 Rajagopalan R, Vaucheret H, Trejo J, Bartel DP. A diverse and evolutionarily fluid set of microRNAs in Arabidopsis thaliana. Genes Dev 2006; 20:34073425.

15 Lewis BP, Burge CB, Bartel DP. Conserved seed pairing, often flanked by adenosines, indicates that thousands of human genes are microRNA targets. Cell 2005; 120:15-20. 